\title{
Automated Characterization of Parallel Application Communication Patterns
}

\author{
Philip C. Roth, Jeremy S. Meredith, Jeffrey S. Vetter \\ Oak Ridge National Laboratory \\ One Bethel Valley Road \\ Oak Ridge, TN 37831 USA \\ \{rothpc,jsmeredith,vetter\}@ornl.gov
}

\begin{abstract}
A concise description of an application's communication pattern is often useful, for example, as an efficient way to communicate application behavior to a system vendor. Several existing performance analysis tools can capture aspects of an application's communication behavior such as which processes communicated with which others and the communications operations they used. However, a human with a high degree of expertise is still required to recognize and characterize common communication idioms within the performance data collected by those tools. To simplify this characterization for non-experts, we have developed an approach for automatically characterizing a MPI application's communication behavior. We use the mpiP profiling tool to collect information about an application's communication topology and message volume. We then use a post-mortem search-based analysis to compare the application's observed communication pattern against a library of common communication patterns. By comparing the result of the various search paths, our approach identifies the combination of patterns that best matches the observed behavior. To evaluate our approach, we applied it to a synthetic example communication matrix and communication matrices obtained from two scientific applications. We determined that our automated approach was highly effective in characterizing the communication patterns represented in the matrices.
\end{abstract}

This manuscript has been authored by UT-Battelle, LLC under Contract No. DE-AC05-00OR22725 with the U.S. Department of Energy. The United States Government retains and the publisher, by accepting the article for publication, acknowledges that the United States Government retains a non-exclusive, paid-up, irrevocable, world-wide license to publish or reproduce the published form of this manuscript, or allow others to do so, for United States Government purposes. The Department of Energy will provide public access to these results of federally sponsored research in accordance with the DOE Public Access Plan (http://energy.gov/downloads/doepublic-access-plan). This research is sponsored by the Office of Advanced Scientific Computing Research in the U.S. Department of Energy.

(C) 2015 Association for Computing Machinery. ACM acknowledges that this contribution was authored or co-authored by an employee, contractor or affiliate of the United States government. As such, the United States Government retains a nonexclusive, royalty-free right to publish or reproduce this article, or to allow others to do so, for Government purposes only.

HPDC'15, June 15-19, 2015, Portland, OR, USA

Copyright (C) 2015 ACM 978-1-4503-3550-8/15/06 ...\$15.00

DOI: http://dx.doi.org/10.1145/2749246.2749278.

\section{Categories and Subject Descriptors}

I.5.0 [Pattern Recognition]: General; D.2.8 [Software Engineering]: Metrics

\section{Keywords}

Application characterization; communication patterns; Message Passing Interface (MPI)

\section{INTRODUCTION}

Contemporary extreme-scale computing systems use a variety of interconnection networks $[30,8,2,1]$ that represent different design choices in terms of performance, manufacturability, reliability, power, and cost. These design points are realized with balanced choices in multiple factors: component integration, optical and electrical communication technologies, and topology complexity. Recent reports $[18,6]$ predict that future system architectures must continue to make tradeoffs in these factors, so it is more important than ever to make judicious choices to ensure that future networks match the requirements of their applications. Clearly, knowing these requirements is a critical first step to this design process, and being able to communicate these requirements among stakeholders greatly increases the likelihood that the final design will truly satisfy application requirements.

In this regard, researchers including application developers, computer architects, and performance engineers need accurate, concise, scalable, and flexible methods and metrics to describe application communication patterns [33, 25, 31]. Without such concise descriptions, architects are typically forced to use overly-simplistic descriptions of communication workloads, or use massive communication trace files on small scale systems to represent application workloads. Producing these concise descriptions is often difficult, however. Tools like the Tuning and Analysis Utilities [26] (TAU), SCALASCA [9], and mpiP [20, 32] capture performance data about an application's communication behavior in event traces or profiles and may even be able to visualize the captured data, but recognizing even common communication patterns in these visualizations requires a high degree of user expertise.

\subsection{Contributions}

To address this challenge, we have developed an approach for automatically characterizing an application's communication behavior. Our approach focuses on HPC applications that use the Message Passing Interface [10, 11] (MPI); we 
use the mpiP performance tool $[32,31]$ to capture information about how an application communicates, including its communication topology and message volume. We, then, use object recognition algorithms to compare the captured data against a library of common communication patterns to identify the pattern (or combination of patterns) that best explains the target application pattern. In addition to identifying these pattern(s), our approach reports a residual metric describing how closely the reported patterns match the observed behavior.

Specifically, the contributions of this work include the following:

- A novel approach for automatically characterizing a MPI application's communication behavior;

- A prototype implementation that works on scalable, real-world applications;

- A thorough discussion of a communication pattern characterization example using a synthetic communication pattern matrix; and

- Case studies of communication pattern characterization of the empirical behavior of two scientific applications.

The primary benefit of this work is in easing the recognition of common communication idioms exhibited in parallel applications, but in doing so we also define a concise language for representing these idioms and combinations thereof.

\subsection{Approach Overview}

Our approach for automatically recognizing parallel application communication patterns is inspired by object recognition strategies used in astronomy. When astronomers seek to identify a new object in the sky, or to see how a known object has moved, they sometimes use a technique called sky subtraction. In this technique, a known pattern is removed from an original image in the hopes that it will be easier to recognize interesting objects in the resulting image. For instance, given an image of the sky in the visual spectrum, an astronomer might remove the ambient background light by subtracting a constant amount of brightness from every pixel in the image. In the resulting image, objects that were only slightly brighter than the ambient background are often more easy to detect than in the original image.

We adopt this sky subtraction idea to recognize common patterns in an application's observed communication data. Starting with a communication matrix $M$ such as the example shown in Figure 1a that contains data about an application's observed message volume and communication pattern, the approach searches for a combination of patterns that best explains the matrix data. In these matrix visualizations, the block at row $i$ and column $j$ is colored according to the amount of data that the process with MPI rank $i$ transferred to the process with rank $j$. The approach attempts to recognize each pattern from its pattern library in the matrix $M$. If a pattern is recognized, such as the $8 \times 8$ two-dimensional nearest neighbor pattern shown in Figure 1b, its contribution is removed from $M$ to produce a matrix $M^{\prime}$ (Figure 1c), and the pattern recognition step is repeated upon $M^{\prime}$. Because multiple patterns might be recognized at each search step, the search produces a results tree such as the one shown in Figure 2 that displays the results of characterizing the matrix from Figure 1a. In this results tree, a matrix is associated with each node and a communication pattern with each edge, such that the pattern associated with the edge between node $N$ and $N^{\prime}$ was recognized in node $N$ 's matrix, and removing the pattern produced node $N^{\prime}$ 's matrix. If no pattern can be recognized in a matrix, its node is a leaf, and the path from the tree root to that leaf defines a collection of patterns that can be removed from the original matrix to produce the leaf's matrix. Once the search is complete (i.e., no more patterns can be recognized in any of the matrices associated with tree nodes), the approach identifies a leaf node whose matrix exhibits the smallest residual value. The patterns along the path from the root to this leaf are the combination of patterns that best explains the original communication matrix. Figure 3 shows the intermediate matrices produced when recognizing and removing the patterns that best explain the Figure 1a matrix. These matrices correspond to the path drawn using thick red lines in the search results tree of Figure 2. Our approach identified six of the seven patterns used to produce the original matrix. The only pattern it did not recognize was the "garbage" pattern we used to place values randomly throughout the communication matrix. We have not yet implemented pattern recognition for this type of random pattern.

\section{BACKGROUND}

Our work on automatically recognizing common communication patterns builds on previous work on MPI performance tools and the characterization of scientific applications.

\subsection{Oxbow}

We recently began a multi-year application characterization activity. Our characterization approach uses a collection of tools we call the Oxbow tool set, and mpiP is one of the tools in this set. In our initial study [31], we characterized the computation and communication behavior of twelve scientific applications and benchmarks. We were especially interested in the proxy applications being developed within the United States Department of Energy (DOE)'s Co-design Centers. When run, a "proxy app" is intended to mimic part (but not all) of a full application that is either not able to be distributed widely or is too expensive to run in its full form. For instance, the Nekbone proxy app is intended to mimic some aspects of the Nek5000 [21] fluid dynamics solver. Whereas Nek5000 is more general, Nekbone only includes a basic conjugate gradient solver. In our initial study, we considered how well the proxy applications represented their associated full applications. More generally, we considered which metrics describing an application's behavior were most useful for characterizing the application, and how to obtain those metrics efficiently.

\section{2 mpiP}

To collect information about an application's communication behavior, we use a modified version of the mpiP [20] lightweight profiling tool. This tool was originally developed to support an approach for analyzing the scalability of an application's communication [32]. The tool is implemented as a library containing functions that are interposed between an application and the MPI library at that library's profiling interface. The tool's versions of MPI functions collect statistics about the application's use of MPI operations 


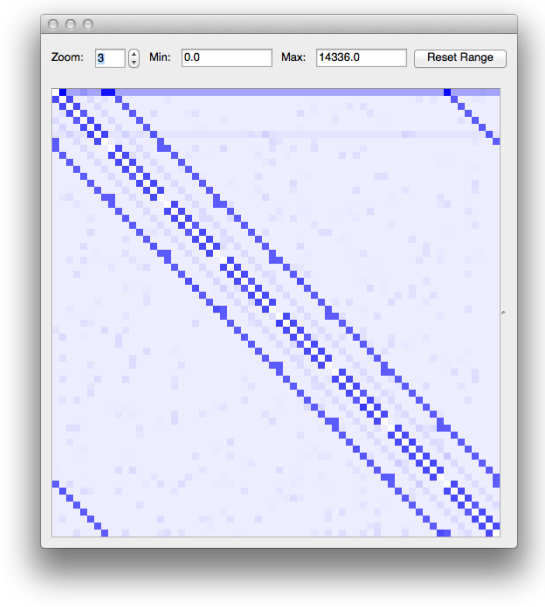

(a) Example communication matrix.

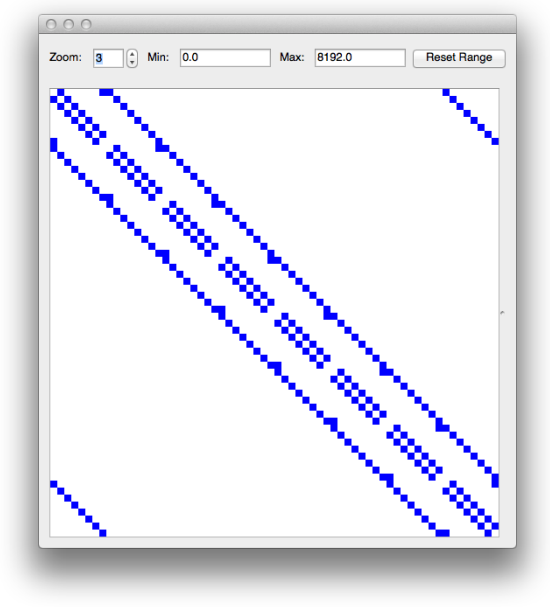

(b) $2 \mathrm{D}$ nearest neighbor pattern recognized in communication matrix.

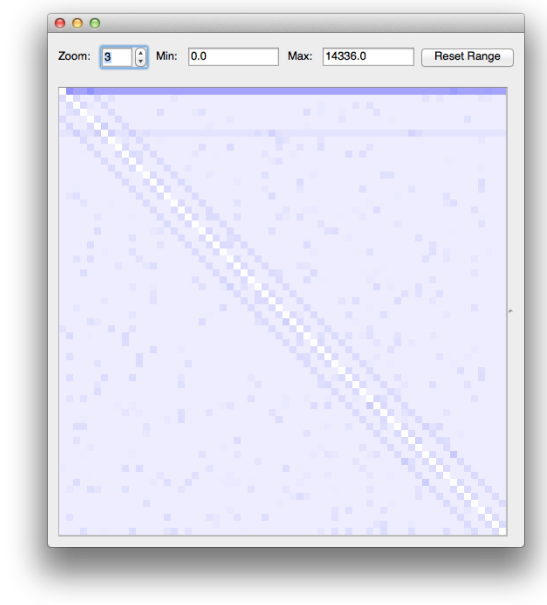

(c) Resulting communication matrix.

Figure 1: Recognizing and removing the contribution of a communication pattern from a communication matrix. The synthetic example communication matrix represents a 64 process run of an application that exhibits several diverse communication patterns. This recognition/removal is only one step in the recognition of the six communication patterns of the original matrix.

that are reported when the application terminates. These reports can also be produced at other times to support collecting performance data for specific phases of the program's execution.

Until recently, mpiP collected and reported statistical data about an application's communication from only a codecentric perspective. The tool tracked how much data was sent and received at each application call site and how long those operations took. This information was useful, but not sufficient, for our Oxbow application characterizations. For the Oxbow study, we modified mpiP so that its MPI interposition functions also track how much data is transferred from each application process to each other process. For example, when the application calls MPI_Send, our version of mpiP tracks not only how long the send operation took and how much data was transferred, but also the destination process. In addition to mpiP's traditional report, our mpiP version also generates several additional output files containing:

1. A matrix indicating the number of times each process sent data to each other process using MPI point-topoint operations;

2. A matrix indicating the number of bytes each process sent to each other process using MPI point-to-point operations;

3. A histogram of the number of bytes involved in each collective communication operation;

4. A matrix indicating the number of bytes each process contributed to MPI collective operations; and

5. A matrix indicating the number of bytes each process received from MPI collective operations.

In file 1 , the value at $(i, j)$ indicates the number of times MPI rank $i$ sent data to MPI rank $j$, and in file 2, the value at $(i, j)$ indicates the amount of data sent from rank $i$ to rank $j$. Taken together, these matrices can be used to compute the average number of bytes per transfer between any pair of processes. Figure 4 shows a visualization produced by VisIt [5] that displays average point-to-point communication volume information collected during a 96-process run of the Large-scale Atomic/Molecular Massively Parallel Simulator [23] (LAMMPS) on the Keeneland Initial Delivery System [29] with the EAM benchmark problem inputs. Also, the matrices in files 2,4 , and 5 can be combined to produce a single matrix that represents the amount of data transferred from each process to the others using any kind of MPI operation. Our automated communication pattern characterization approach can work on each file individual, or on the combined matrix as illustrated in Section 4. The count and volume information that mpiP represents using a matrix can also be represented as a directed graph whose vertices represent application processes and whose edges represent data transfers between processes. Because the application's communication pattern can be represented using these graphs, this data may be said to express the application's communication topology.

\section{AUTOMATIC CHARACTERIZATION OF COMMUNICATION PATTERNS}

Algorithm 1 shows the pseudocode for our approach for automatic characterization of application communication patterns. As presented here, the algorithm operates on a message volume matrix, but the algorithm is nearly identical when operating on an operation count matrix. For input, the algorithm takes a communication matrix $M$ and a library $L$ of communication patterns. The algorithm outputs the set of patterns and scaling factors that best account for the observed communication topology and message volume data in $M$, or a message indicating that the algorithm could 


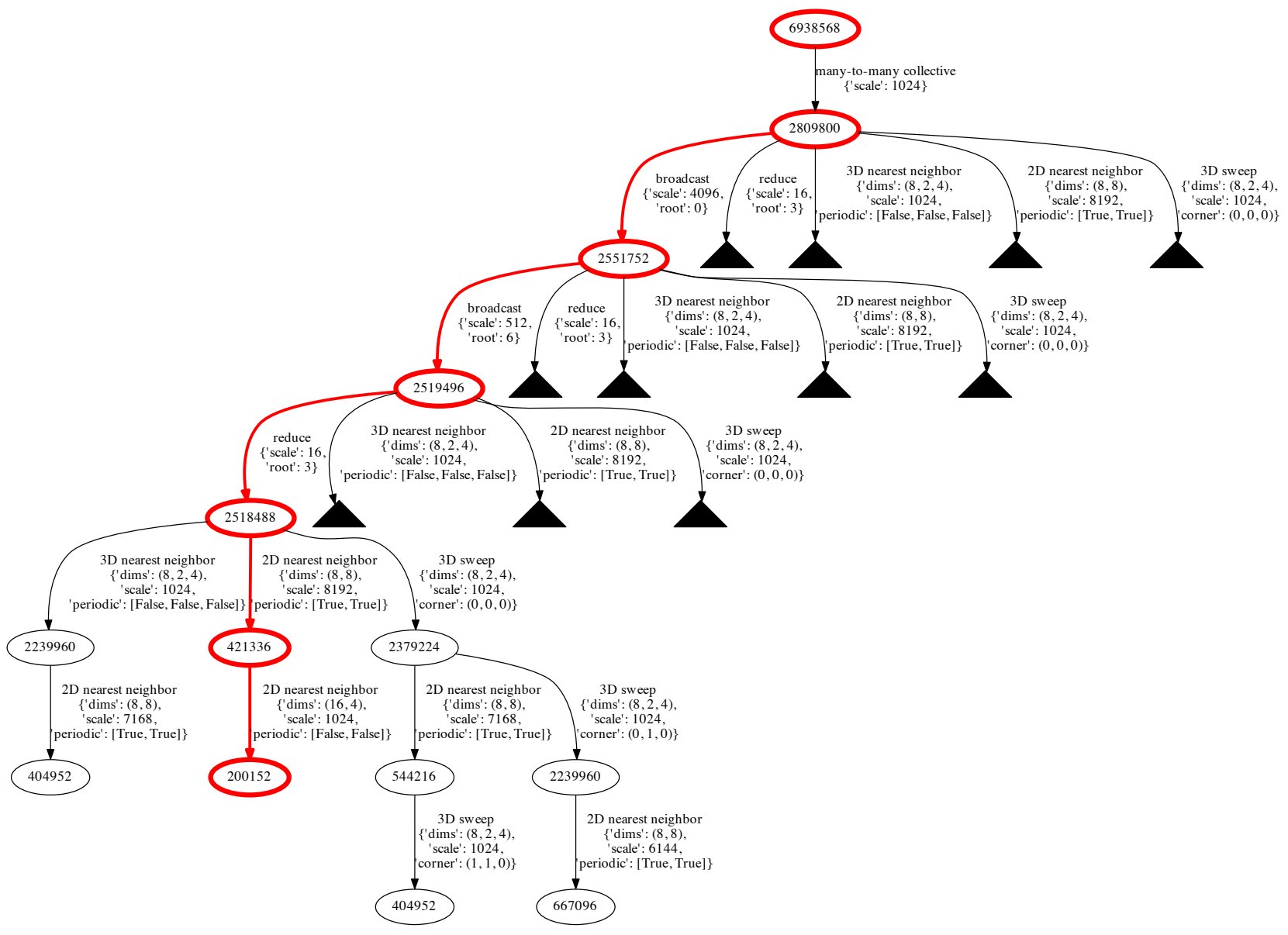

Figure 2: Pattern recognition results for the example matrix shown in Figure 1a. Each node is labeled with the residual metric computed for the matrix associated with that node. Each edge is labeled with the pattern recognized within the parent

node's matrix and removed to produce the child node's matrix. Black triangles indicate subtrees elided due to space constraints. Nodes and edges drawn in thick red lines indicate the combination of patterns that best explain the original communication matrix data.

not identify any of its known patterns in the input matrix. In this section, we describe the algorithm and our initial implementation of it in detail.

First, the algorithm uses search to determine which combinations of patterns it can recognize in the input matrix, maintaining the results of this search in a tree. It creates a tree root node for the input matrix (line 3 ) and calls the recursive Refine function on that root node to complete the search (line 4). Given a node, the Refine function (lines 17-30) attempts to recognize patterns in that node's matrix. If a pattern is recognized, the Refine function generates a "pure" pattern matrix (line 22). This pattern matrix is the matrix that would result if an application only exhibited the recognized pattern. Pattern recognition and pattern matrix generation is described in more detail in Section 3.1. The Refine function then subtracts the pattern matrix from the node's matrix (line 23), and the resulting matrix is associated with a new node (line 24) that will become a child of the current node. The child node is attached to the parent node using an edge, to which the recognized pattern is asso- ciated (lines 25-26). Finally, the Refine function is called recursively on the new child node. Our prototype implementation of the search (see Section 3.4) is sequential, but because there are no dependencies between the search refinements rooted at each child, the search could be parallelized.

After building the search results tree, the algorithm traverses the tree to identify the tree leaf node whose associated matrix has the smallest residual (line 5; the FindSmallestResidual function is not presented here due to space limitations). This residual metric is discussed further in Section 3.2. Once a leaf with the smallest residual is identified, the algorithm walks the path from the leaf to the search results tree's root, collecting the recognized patterns from the edges along this path (lines 7-13) into a set that it outputs as the algorithm's result (line 14). Note that the patterns in this set can be removed from the original communication matrix in any order to produce the matrix with the minimal residual. 


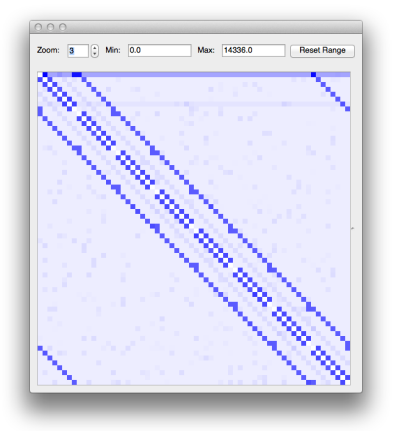

(a) Synthetic communication matrix.

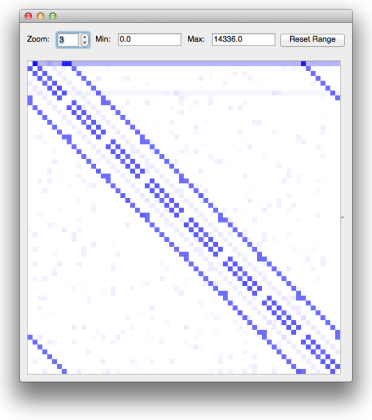

(b) Matrix after removing many-to-many pattern, scale:1024.

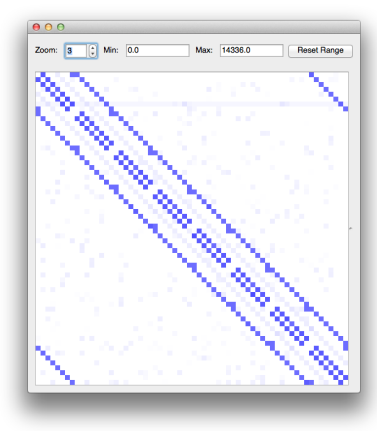

(c) Matrix after removing broadcast pattern, scale: 4096, root: 0 .

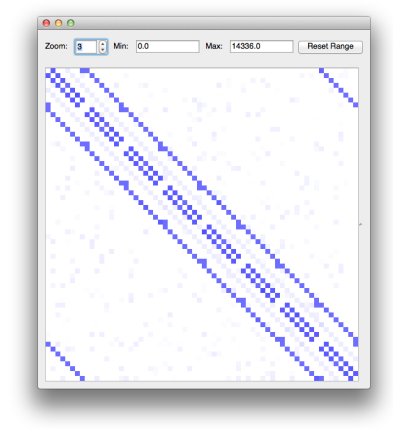

(d) Matrix after removing broadcast pattern, scale: 512 , root: 6 .

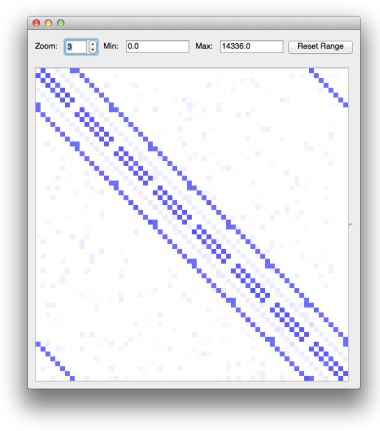

(e) Matrix after removing reduce pattern, scale: 16 , root: 3 .

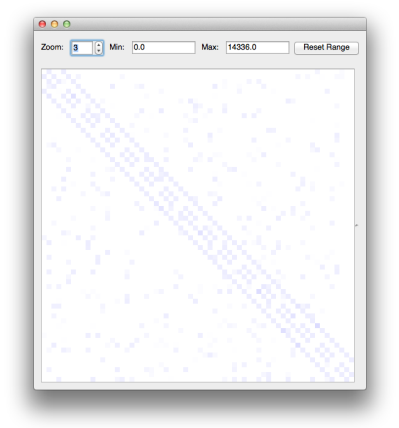

(f) Matrix after removing 2D nearest neighbor pattern, dims: $(8,8)$, scale: 8192 , periodic.

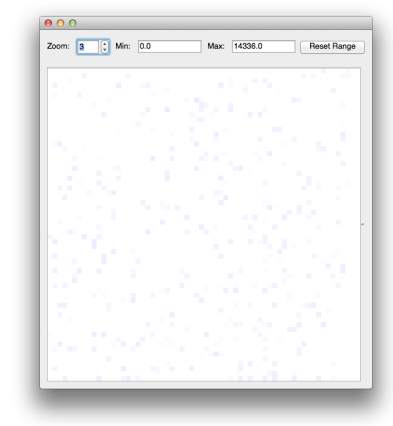

(g) Final matrix after removing $2 \mathrm{D}$ nearest neighbor pattern, dims: $(16,4)$, scale: 1024 , not periodic.

Figure 3: Sequence of recognized patterns and intermediate matrices for a synthetic communication matrix.

\subsection{Patterns}

Our automated communication pattern characterization approach uses a library of common communication patterns. For each pattern, the library entry contains the following information:

- Its name.

- A function Recognize(M) that attempts to recognize the pattern in the matrix $M$. If the pattern is recognized, the function returns the pattern's parameters. The parameters returned depend on the pattern. For instance, the Broadcast pattern returns the MPI rank of the Broadcast operation's root process and the amount of data that was broadcast. In contrast, the 2D Nearest Neighbor pattern returns the dimensions of the 2D Cartesian topology the application used, whether the application used periodic boundaries, and the amount of data transferred between pairs of processes. If the pattern is not recognized in the given matrix, this function returns an empty pattern parameter set.

- A function Generate (n, pattern_params) that generates a communication matrix that represents the "pure" pattern for $n$ processes and the given pattern_params. It is this pure pattern matrix that is subtracted from a search results tree node's matrix when a pattern is recognized within the Refine function.

The pattern library uses generator functions so that a pattern description is independent of the number of processes used to run the application. The collection of pattern names in the library define a language that can be used to describe the communication behavior of an application, in much the same way as the "Berkeley Dwarfs" [3] can be used to describe the algorithmic behavior of an application.

The approach used for recognizing a pattern varies depending on the pattern. Recognizing some patterns is fairly easy. For instance, the broadcast pattern's Recognize function looks for a row in the matrix $M$ whose elements are all non-zero, except for the element on $M$ 's diagonal. If the function identifies such a row, it indicates the row number as the broadcast operation's root, and the minimum non-zero value of the row as the operation's scale-the amount of data broadcast. The pattern uses the minimum non-zero value as the scale because the scaled pure pattern, when removed from $M$, would produce a matrix $M^{\prime}$ with negative values that are invalid given our definition of a communication matrix.

Other patterns are more challenging to recognize. For example, the 2D nearest neighbor pattern's Recognize function looks for the following criteria in the input matrix $M$ : 

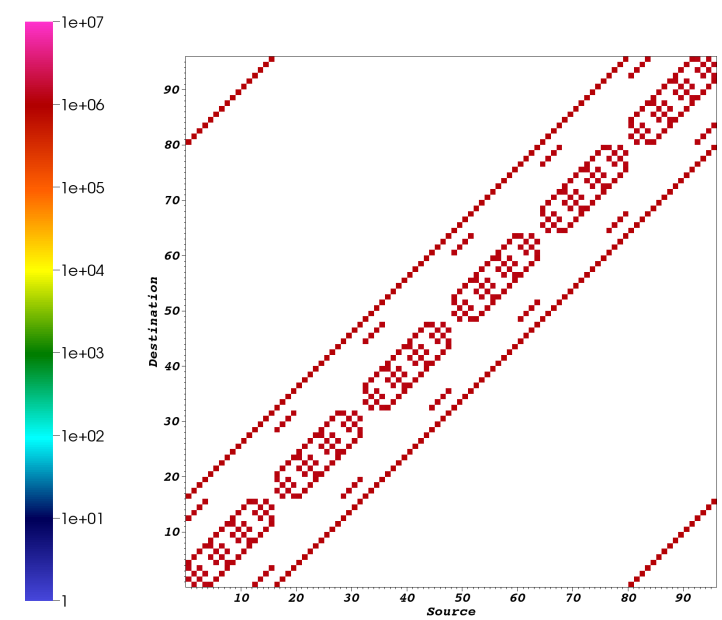

Figure 4: Average volume of point-to-point communication for LAMMPS, EAM benchmark problem, with 96 processes.

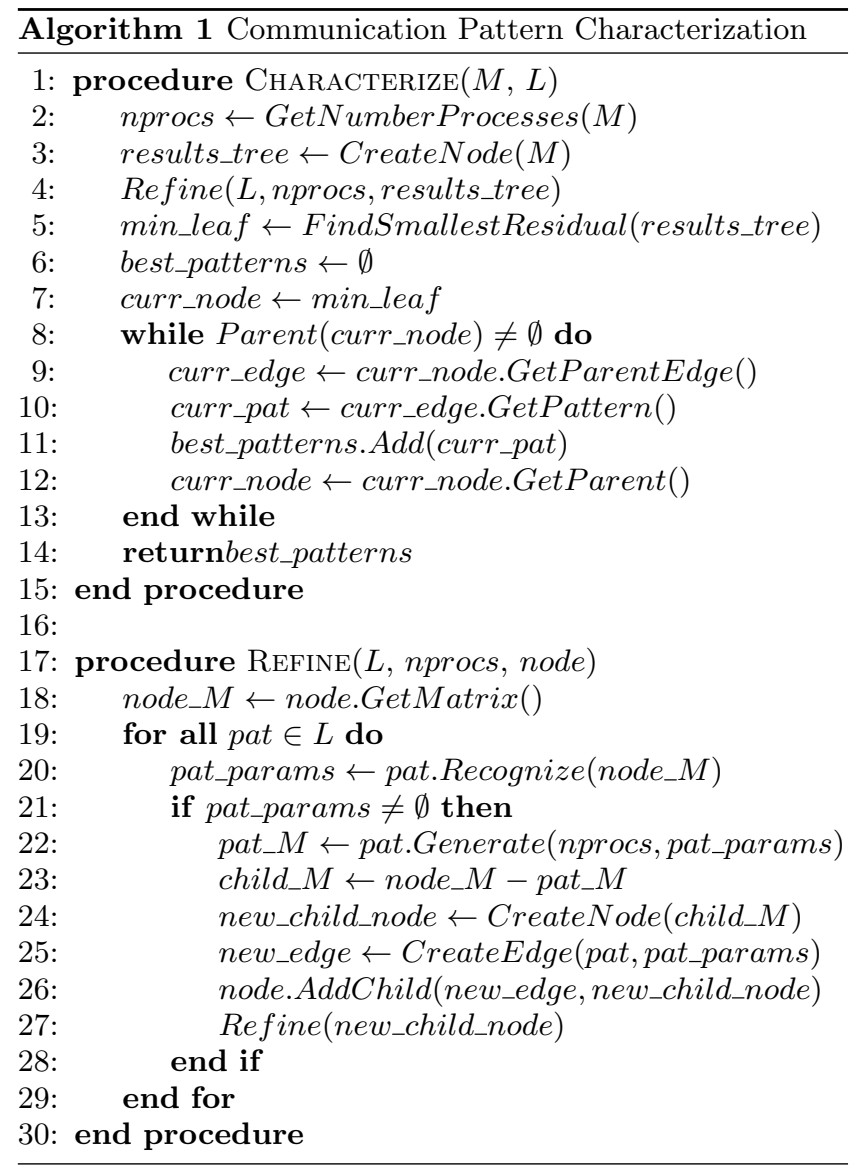

- The \pm 1 diagonals (i.e., the diagonals just above and just below the matrix's main diagonal) are mostly nonzeros, but not entirely.

- There is a pair of $\pm x$ diagonals that are entirely nonzeros.

- The $x$ identified by the previous criteria divides the number of processes evenly. This $x$ is the number of columns in the 2D Cartesian topology, and nprocs/x is the number of rows.

- If the $\pm($ nprocs $-x)$ diagonals are entirely non-zeros, the pattern uses periodic boundaries.

Some of these criteria are intentionally vague, such as the requirement for diagonals to be mostly non-zeros, because they are used as lightweight checks to rule out the pattern as early as possible. These lightweight criteria also provide potential values for the pattern's parameters, such as the dimensions of the 2D Cartesian topology used. If all of these lightweight criteria are satisfied, the Recognize function uses these potential parameters and the pattern's Generate function to produce a pure pattern matrix to be used as a mask against $M$. For every non-zero value in the mask, Recognize checks whether the value at the same (row, column) coordinates in $M$ is also non-zero. During this check, the Recognize function also tracks the minimum non-zero masked element in $M$, to be used as the pattern's scale if it is recognized successfully. If and only if all non-zero elements in the mask are also non-zero in $M$, the Recognize function returns the recognized dimensions, scale, and boundary periodicity as the pattern parameters.

\subsection{Residual Metric}

Algorithm 1 determines whether one collection of recognized patterns is better than another by comparing a residual metric computed for the resulting communication matrices. When computed for a matrix, this residual metric must indicate the amount of data that is not yet accounted for using known communication patterns. For our prototype implementation of Algorithm 1, we use a simple definition of the residual metric: the total data volume indicated by the matrix (i.e., the sum of the matrix elements). As evidenced by Figure 2, the values of this residual metric can be 
quite large even for application runs with a modest number of processes, so to improve the user-friendliness of our implementation's interface these residual values may be expressed as percentages of the original matrix's residual value.

\subsection{Recognition Order}

The order in which patterns are recognized matters, both in terms of the quality of the algorithm's results and as a performance optimization. The approach attempts to recognize each pattern during each search step because the recognition and removal of one pattern's contribution can preclude the recognition of another pattern later. For example, the synthetic example matrix shown in Figure 1a was constructed by combining seven communication patterns, including two different 2D nearest neighbor patterns. However, the combination of these patterns also matches a $3 \mathrm{D}$ nearest neighbor pattern. If the characterization algorithm recognizes and removes the 3D pattern first, the algorithm is no longer able to recognize a $2 \mathrm{D}$ pattern in the resulting matrix. On the other hand, if it recognizes a $2 \mathrm{D}$ pattern first, it is able to recognize the second $2 \mathrm{D}$ pattern. The residual metric of the matrix produced by recognizing both $2 \mathrm{D}$ patterns is smaller than that of the matrix produced by recognizing only the 3D pattern. Situations like this are the reason our algorithm attempts to recognize all patterns at each step in the search and compares the residual metric for the resulting methods only when the algorithm can no longer recognize further patterns along each search path.

Although recognizing a pattern $P_{1}$ instead of pattern $P_{2}$ may preclude later recognition of pattern $P_{3}$ as described in the example above, if both $P_{1}$ and $P_{2}$ are recognized, it makes no difference in which order they are recognized ( $P_{1}$ then $P_{2}$ or vice versa). The algorithm as shown in Algorithm 1 does not attempt to optimize the search by eliminating search paths that represent permutations of paths that have already been searched, and explains why the search results tree shown in Figure 2 contains several paths that are permutations of each other. We leave this performance optimization for future work.

The quality and performance of the search are also affected in situations where a given pattern can be recognized in multiple ways. For example, if the communication matrix $M$ includes an all-to-all communication pattern (called "many-to-many" in our prototype implementation), it is equally valid to recognize the pattern as one many-tomany operation, the combination of nprocs broadcast operations each rooted at a different application process, or the combination of nprocs reduce operations. The communication matrix itself does not contain enough information to determine which of these approaches were actually used in the application. However, recognizing this pattern as nprocs broadcast or reduce operations greatly increases the amount of time required to search for pattern combinations, and greatly increases the number of constituent patterns output by the algorithm, so we prefer to recognize and remove the many-to-many pattern first. Although line 19 of Algorithm 1 does not indicate that the Refine function attempts to recognize patterns in any order, our prototype implementation attempts to recognize the many-to-many pattern first. This is why the many-to-many pattern was recognized first in the search results shown in Figure 2.

\subsection{Implementation}

We have implemented our automated communication characterization algorithm in a Python command line tool called AChax (with pronunciation similar to "Ajax"), for Automated Characterization. Each pattern is implemented as a Python class with instance methods Recognize, and Generate. To date, we have implemented the classes for the following patterns:

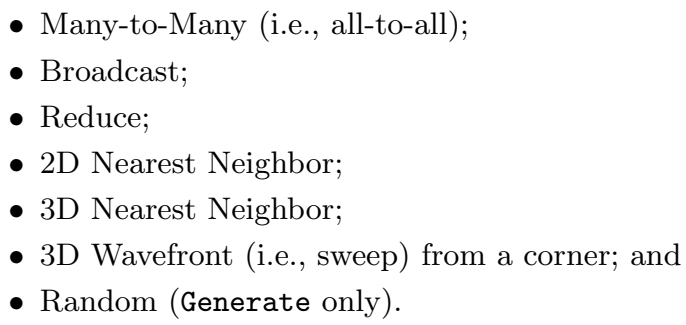

A class named AChaxer implements Algorithm 1. The program's singleton instance of this class builds its pattern library by creating an instance of each of the known pattern classes, and placing them in a Python list. The AChaxer object's implementation of the Refine method attempts to recognize the patterns in the order they appear in this list, so as described in Section 3.3, the many-to-many pattern instance is placed first in the list. The AChaxer object's Characterize method uses its Refine method to build the search results tree, and implements the FindSmallestResidual method to identify the leaf node whose matrix has the smallest residual metric value. Although the ordering of the patterns recognized to produce this smallest residual matrix does not matter, to support debugging the AChaxer object reverses the order obtained by walking from leaf to root before outputting its results, so that the output matches the order in which the patterns were actually recognized. The AChaxer object can optionally write output files that contain:

- The matrix identified as having the smallest residual;

- A log of the actions performed during the automated search, including which patterns were tried and the pattern parameters of recognized patterns;

- A DOT representation of the search results graph that can be visualized using the Graphviz dot command; and

- A sequence of matrices showing the intermediate result of removing each recognized pattern until achieving the matrix with minimal residual.

An AChaxApp object implements the user interface to the tool. This object parses the command line, reads the input communication matrix, and outputs results. The NumPy [27] and SciPy [28] Python packages are used to implement matrix operations including the reading and writing of matrices to files, and both dense and sparse matrix representations are supported and used internally by the tool. Although this implementation is serial and the algorithm performs redundant work as described earlier in this section, the performance of this prototype implementation is still satisfactory. For instance, characterizing the communication patterns from a 216-process application run (detailed in Section 4.3) took less than 90 seconds on a four-year-old Macintosh laptop with $2.66 \mathrm{GHz}$ dual core with $4 \mathrm{~GB}$ memory. 


\section{CASE STUDIES}

\subsection{Test System}

We used the Keeneland Initial Delivery System [29] (KIDS) for our case studies. KIDS is a Georgia Institute of Technology cluster deployed at Oak Ridge National Laboratory. The system contained 120 HP ProLiant SL390 G7 compute nodes. Each compute node contained 24 GB memory, two Intel Xeon X5660 processors running at $2.80 \mathrm{GHz}$, and three NVIDIA M2090 GPUs. The nodes were connected with an Infiniband QDR interconnection network. The system used the CentOS 6.3 Linux distribution on its compute nodes. We used the Intel Composer XE 2013 SP1.1.106 (also reported as version 14.0.1) compilers to build and run the test applications, and OpenMPI 1.6.1 as the MPI library and runtime.

\subsection{LAMMPS}

LAMMPS is a molecular dynamics simulator, written in $\mathrm{C}++$, that uses MPI for interprocess communication and synchronization. We obtained the LAMMPS source code from the project's Git repository, and used revision 42bb280c dated 2014-04-15. We modified the LAMMPS makefile to build on KIDS, and to link in our version of the mpiP library that produces communication topology matrix files. We ran LAMMPS with the EAM benchmark problem input file using 96 processes in a $4 \times 4 \times 63 \mathrm{D}$ Cartesian process topology.

When solving the EAM benchmark problem, LAMMPS uses MPI point-to-point operations in a 3D nearest neighbor communication pattern, and the MPI broadcast, allreduce, and scan collective operations. The broadcast operations are all rooted at MPI rank 0 . The version of mpiP we used for this study models the rootless MPI allreduce operation as a reduce operation to rank 0 , followed by a broadcast from 0 to all other operations. It also models the scan operation as a gather operation of data from all processes to rank 0 , which then computes the scan result and scatters the result to all processes. This may not be how the underlying MPI implements these collective operations, but because mpiP operates at the MPI profiling interface, it has no information about the underlying implementation.

Figure 5 shows visualizations of the communication matrix produced by mpiP for the 96-process LAMMPS run, the patterns recognized by AChax in this matrix, and the matrices produced by removing those patterns. To expose detail that would be hidden if the blue saturation color map of Figure 3 were used, this figure uses a heat map color palette with "hotter" colors (e.g., yellow, orange) indicating larger values and "cooler" colors (e.g., blue, purple) indicating smaller values. Zero values in the communication matrix are indicated using white blocks. As shown in the figure, AChax recognized the 3D nearest neighbor communication pattern, including the correct dimensions of the 3D Cartesion topology used. Because of the way mpiP models MPI_Scan and MPI_Allreduce, AChax cannot distinguish between these operations and MPI_Bcast and MPI_Reduce, and has recognized the communication as the latter pair of patterns. Lacking more information about how the MPI library implements its rootless communication operations, and having mpiP expose that information, the resulting patterns reported by AChax are equivalent as far as their usefulness. We can express the LAMMPS communication be- havior using the following expression, using the scale of each recognized pattern as a coefficient:

$$
\begin{array}{r}
C_{L A M M P S}=13354 \cdot \text { Broadcast }(\text { root }: 0)+ \\
700 \cdot \text { Reduce }(\text { root }: 0)+ \\
19318888 \cdot 3 \text { DNearest Neighbor }( \\
\text { dims }:(4,4,6), \\
\text { periodic }: \text { True })
\end{array}
$$

The error in this expression, visualized as a communication matrix, is shown in Figure $5 \mathrm{~d}$.

At first glance, the residual matrix produced by removing all recognized patterns (Figure 5d) makes it appear as if AChax did not correctly determine the scale of the 3D nearest neighbor pattern, because the residual pattern appears to match the pure 3D nearest neighbor pattern. In fact, AChax did recognize the scale correctly: after removing the recognized pattern, there is a zero element (circled in the figure) in one of the diagonals that must be non-zero for a $3 \mathrm{D}$ nearest neighbor pattern. The residual matrix produced by AChax after removing recognized patterns provides the interesting insight that not only does LAMMPS use a 3D nearest neighbor communication pattern, the amount of data LAMMPS communicates between neighbors varies. The coloration of Figure $5 \mathrm{~d}$ indicates that for the input problem we used, the LAMMPS nearest neighbor communication transferred more data in some dimensions than others. More data was sent by process with rank $i$ to its neighbors with rank $i \pm 1$ (yellow blocks in the figure) than to its neighbors along the next dimension (blue blocks in the figure), and that more than to its neighbors along the final dimension (purple blocks in the figure). Furthermore, the amount of data sent by each proces to its neighbor along that third dimension varies, as indicated by the fact that removing the recognized pattern with its constant scale caused only one of the would-be-purple blocks to have a zero value. If all processes communicated the same amount along this dimension, the resulting matrix would have no non-zeros in these diagonals, and the purple-colored blocks in Figure $5 \mathrm{~d}$ would not be there.

\subsection{LULESH}

The Livermore Unstructured Lagrangian Explicit Shock Hydrodynamics application [13] (LULESH) is a proxy application meant to approximate a typical hydrodynamics model such as ALE3D [22]. LULESH is one of the applications being used for hardware/software co-design within the U.S. Department of Energy's Exascale Co-Design Center for Materials in Extreme Environments [7]. Unlike a full application, LULESH solves a specific, hard-coded problem. We used LULESH version 2.0.3 [14]. This version is written in $\mathrm{C}++$ and can be built for serial execution or parallel execution using MPI or MPI+OpenMP. We ran LULESH on KIDS with 216 processes in a $6 \times 6 \times 63 \mathrm{D}$ process topology.

LULESH uses a limited number of MPI communication operations: non-blocking point-to-point sends and receives, and the reduce and allreduce collective operations. Nevertheless, LULESH exhibits interesting communication patterns for AChax to characterize.

Figure 6 shows visualizations of the communication matrix produced by mpiP for the 216-process LULESH run, the patterns recognized by AChax in this matrix, and the intermediate matrices produced by removing the recognized 


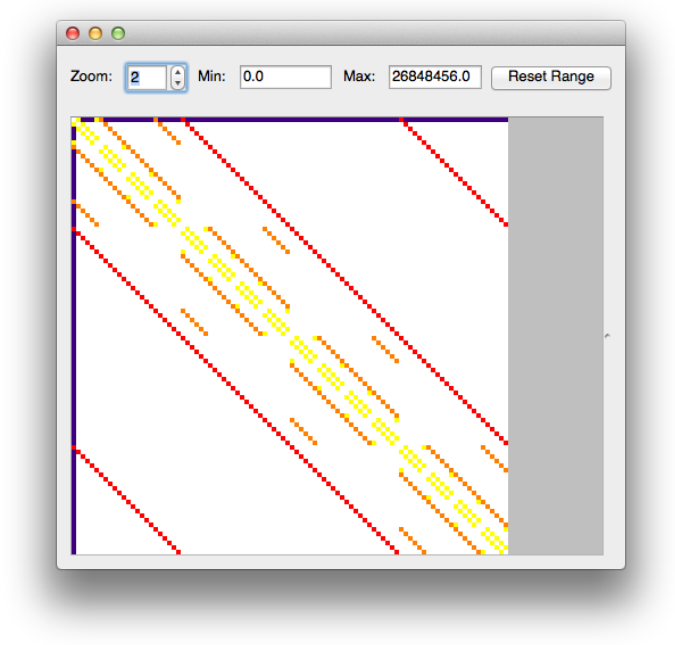

(a) Communication matrix produced by mpiP.

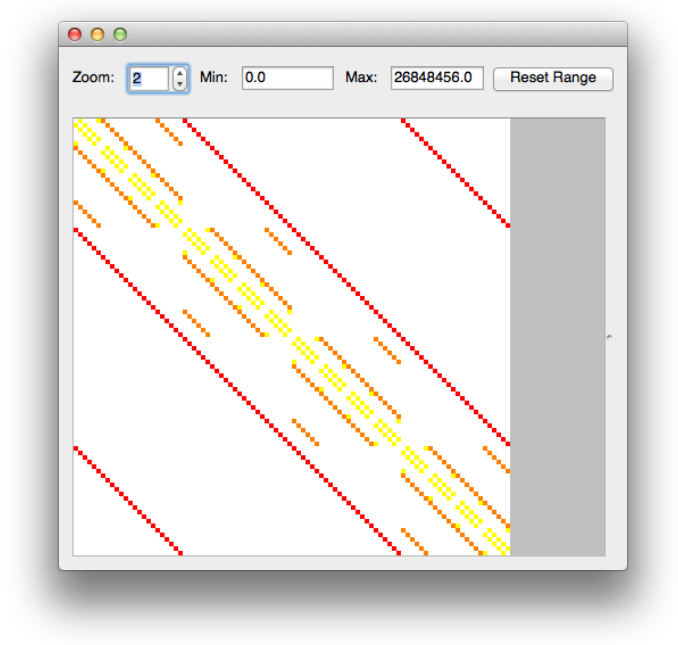

(c) Matrix after removing reduce, root: 0, scale: 700 .

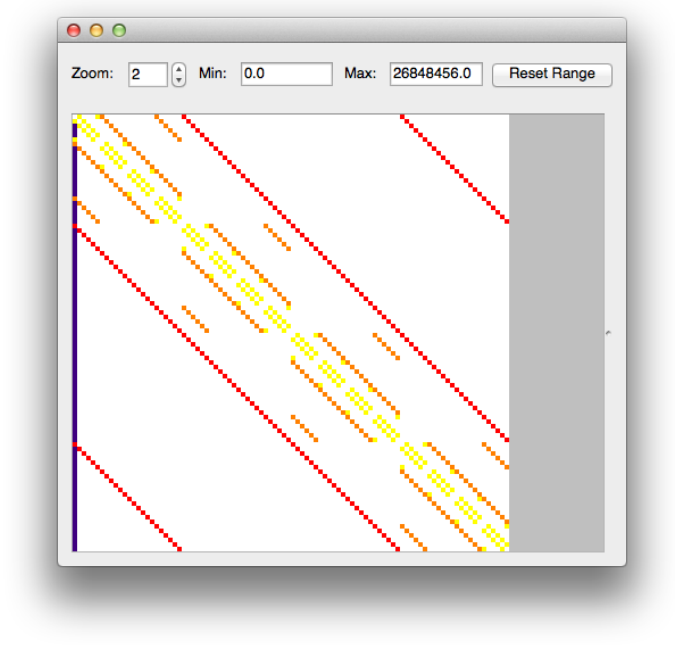

(b) Matrix after removing broadcast, root: 0 , scale: 13354.

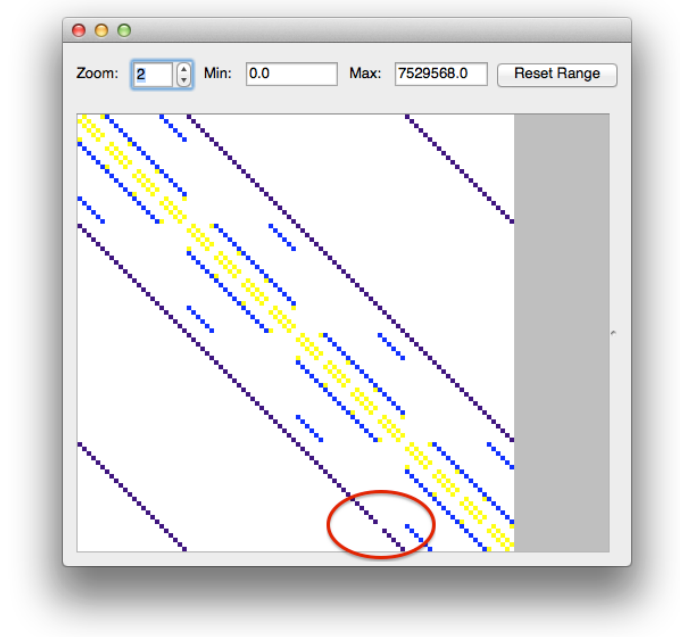

(d) Matrix after removing 3D nearest neighbor, dims: $(4,4,6)$, scale: 19318888 , periodic: True.

Note the different scale compared to the other matrices in the figure, and the zero value indicated with a red oval that precludes the recognition of a $3 \mathrm{D}$ nearest neighbor pattern in this matrix.

Figure 5: Initial communication matrix produced by mpiP, and intermediate and final communication matrices produced by AChax for a 96-process LAMMPS run solving the EAM benchmark problem.

patterns. As with Figure 5 showing LAMMPS communication matrices, this figure uses a heat map color pallette with white blocks indicating zero values in the underlying matrix. As shown in the figure, AChax recognized 3D nearest neighbor and 3D sweep patterns with the correct Cartesian process topology dimensions, and recognized LULESH's use of Allreduce as combinations of broadcast and reduce as described in Section 4.2. The AChax-characterized expression for LULESH's communication pattern is:

$$
\begin{array}{r}
C_{\text {LULESH }}=51984 \cdot \text { Broadcast }(\text { root }: 0)+ \\
51992 \cdot \text { Reduce }(\text { root }: 0)+ \\
290279024 \cdot 3 \text { DNearestNeighbor }( \\
\text { dims }:(6,6,6), \\
\text { periodic }: \text { False })+ \\
299785872 \cdot 3 \text { D Sweep }( \\
\text { dims }:(6,6,6), \\
\text { corner }:(5,5,5))
\end{array}
$$




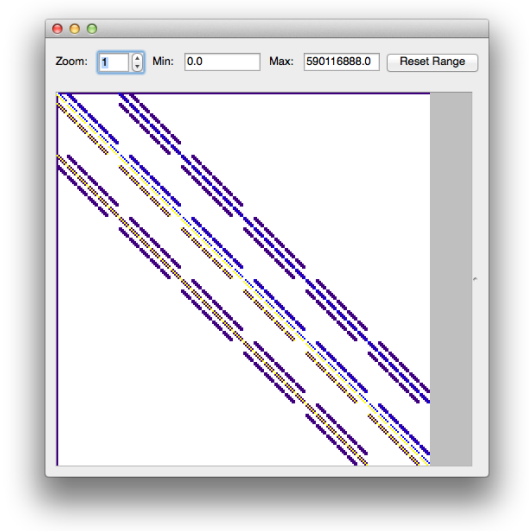

(a) Communication matrix produced by mpiP.

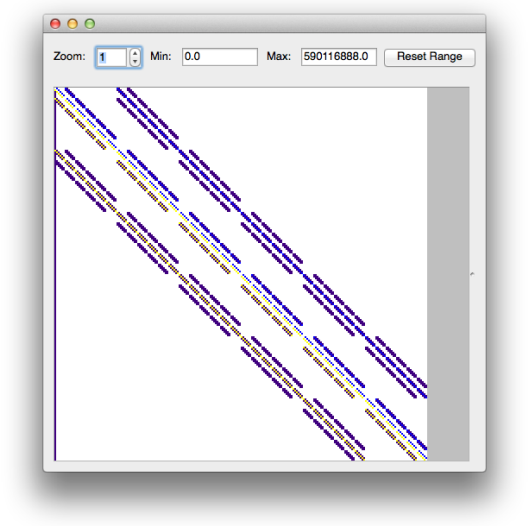

(b) Matrix after removing broadcast, root: 0, scale: 51984 .

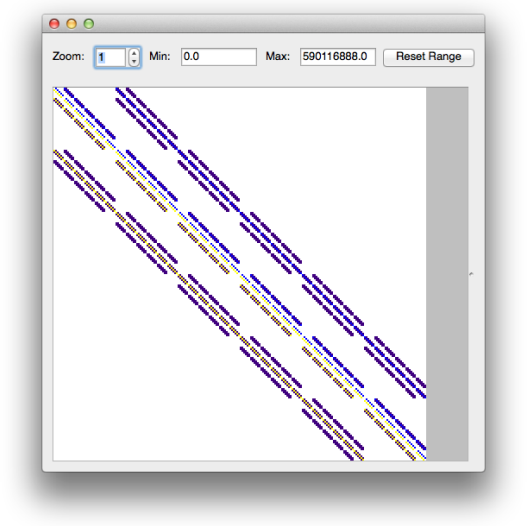

(c) Matrix after removing reduce, root: 0, scale: 51992 .

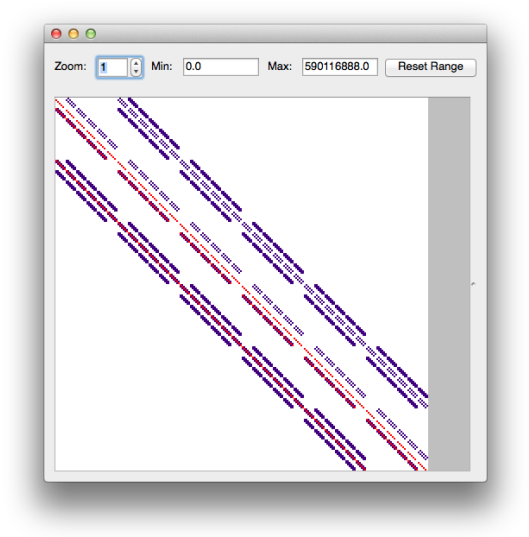

(d) Matrix after removing 3D nearest neighbor, dims: $(6,6,6)$, scale: 290279024, periodic: False.

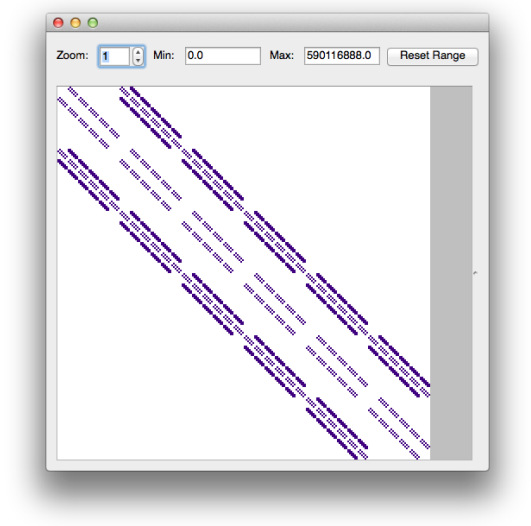

(e) Matrix after removing 3D sweep, dims: $(6,6,6)$, scale: 299785872 originating corner: $(5,5,5)$.

Figure 6: Initial communication matrix produced by mpiP, and intermediate and final communication matrices produced by AChax for a 216-process LULESH run.

Interestingly, the residual matrix (Figure 6e) exhibits a communication pattern for which there is not yet a pattern in AChax's pattern library. The pattern is regular and has hallmarks of a near-neighbor (but not necessarily nearest) pattern. The AChax pattern library is not considered complete, and identifying this pattern and adding a pattern recognizer and generator for it is one of our near-term future AChax work activities.

\section{RELATED WORK}

Researchers have long recognized the need to characterize communication patterns. For example, Kim and Lilja [17] characterized the communication patterns of several message passing applications and benchmarks from the NAS Parallel Benchmark suite, to study whether the benchmarks' behavior was similar to that of the applications. Karlsson and Brorsson [15] performed a similar study of a few applications to contrast their communication behavior when implemented using message passing and shared memory. More recently, Raponi et al [24] performed a similar study of applica- tions and benchmarks from the Sequoia benchmark suite and from Argonne National Laboratory's workload. Although each of these studies produced useful information about the communication behavior of scientific applications, none used automated methods to characterize the communication behavior.

The communication pattern characterization approach that is most similar to ours is described by Kerbyson and Barker [16]. Both approaches perform automated characterization of application communication patterns by matching observed communication data against a library of known communication patterns. Both approaches dynamically produce a pattern mask and compare it to the observed communication data when attempting to match a pattern, and neither approach has support (yet) for identifying the patterns produced when a subset of all application processes communicate. Nevertheless, there are several substantial differences between the two approaches. Whereas their work considers only point-to-point communication, ours supports patterns produced using both point-to-point and collective operations. Their approach indicates only that a pattern was matched, 
whereas ours identifies how much data was transferred for each matched pattern. Their approach matches patterns on a per-call-site basis, and requires manual application instrumentation to identify those call sites. In contrast, our approach uses an automated search through a pattern space to identify the combination of patterns that best explains the application's communication behavior. Our approach does not require source code modification, but the mpiP application programming interface can be used to limit the collected communication data, and thus the communication pattern characterization, to a specific application phase or even a specific call site if desired.

Our approach draws on techniques used in automated performance analysis. For example, Miller et al [19] presented a search-based approach for automatically analyzing the computation and communication behavior of a running application to identify performance bottlenecks. Bhatia et al [4] presented an automatic analysis of application communication behavior, but their analysis sought to identify the cause of communication performance problems rather than to characterize the communication patterns used by the application. Unlike our work whose goal is to characterize application behavior (specifically communication behavior), this earlier research focuses on the complementary goal of automatically identifying the causes of application performance problems. He et al [12] presented an automated compiler-based approach for recognizing performance idioms, or common scientific computing patterns, in several of the NAS Parallel Benchmark suite. Although that work suggests detection of idioms that include both computation and communication, the paper provides no detail about recognition of communication patterns, whereas that is the focus of the work presented in this paper.

\section{SUMMARY}

In this paper, we described a novel approach for automatically characterizing a parallel application's communication behavior. Our approach uses a search algorithm with a library of known communication patterns to identify the pattern, or combination of patterns, that best explain the application's communication behavior. AChax, our implementation of the approach, applies our characterization algorithm on files produced by mpiP that contain information about an application's topology and message volume in the form of a communication matrix. In case studies using a complex synthetic communication matrix and matrices captured from the LAMMPS and LULESH scientific applications, we demonstrated the efficacy of our approach in automatically identifying the applications' communication patterns.

Going forward, we plan to improve the efficacy of our automated characterization approach by implementing support for the recognition of more types of communication patterns such as wavefront patterns, by researching whether image recognition techniques can improve or simplify pattern recognition, and by adding support for applications that use sub-communicators. We also plan to improve the performance of our search by implementing techniques for pruning the search when it determines it is attempting to search a permutation of patterns that have already been attempted, and by parallelizing the search.

\section{REFERENCES}

[1] Y. Ajima, S. Sumimoto, and T. Shimizu. Tofu: A 6D mesh/torus interconnect for exascale computers. IEEE Computer, 42(11):36-40, 2009.

[2] B. Arimilli, R. Arimilli, V. Chung, S. Clark, W. Denzel, B. Drerup, T. Hoefler, J. Joyner, J. Lewis, L. Jian, N. Nan, and R. Rajamony. The PERCS high-performance interconnect. In High Performance Interconnects (HOTI), 2010 IEEE 18th Annual Symposium on, pages 75-82, 2010.

[3] K. Asanovic, R. Bodik, B. C. Catanzaro, J. J. Gebis, P. Husbands, K. Keutzer, D. A. Patterson, W. L. Plishker, J. Shalf, S. W. Williams, and K. A. Yelick. The landscape of parallel computing research: A view from berkeley. Technical Report UCB/EECS-2006-183, EECS Department, University of California, Berkeley, Dec 2006.

[4] N. Bhatia, F. Song, F. Wolf, J. Dongarra, B. Mohr, and S. Moore. Automatic experimental analysis of communication patterns in virtual topologies. In Parallel Processing, 2005. ICPP 2005. International Conference on, pages 465-472, June 2005.

[5] H. Childs, E. Brugger, B. Whitlock, J. Meredith, S. Ahern, D. Pugmire, K. Biagas, M. Miller, G. H. Weber, H. Krishnan, T. Fogal, A. Sanderson, C. Garth, E. W. Bethel, D. Camp, O. Rübel, M. Durant, J. Favre, and P. Navratil. VisIt: An end-user tool for visualizing and analyzing very large data. In E. W. Bethel, H. Childs, and C. Hansen, editors, High Performance Visualization-Enabling Extreme-Scale Scientific Insight, Chapman \& Hall, CRC Computational Science, pages 357-372. CRC Press/Francis-Taylor Group, Boca Raton, FL, USA, Nov. 2012. http:

//www.crcpress . com/product/isbn/9781439875728, LBNL-6320E.

[6] J. Dongarra, P. Beckman, T. Moore, P. Aerts, G. Aloisio, J.-C. Andre, D. Barkai, J.-Y. Berthou, T. Boku, B. Braunschweig, F. Cappello, B. Chapman, X. Chi, A. Choudhary, S. Dosanjh, T. Dunning, S. Fiore, A. Geist, B. Gropp, R. Harrison, M. Hereld, M. Heroux, A. Hoisie, K. Hotta, Z. Jin, Y. Ishikawa, F. Johnson, S. Kale, R. Kenway, D. Keyes, B. Kramer, J. Labarta, A. Lichnewsky, T. Lippert, B. Lucas, B. Maccabe, S. Matsuoka, P. Messina, P. Michielse, B. Mohr, M. S. Mueller, W. E. Nagel, H. Nakashima, M. E. Papka, D. Reed, M. Sato, E. Seidel, J. Shalf, D. Skinner, M. Snir, T. Sterling, R. Stevens, F. Streitz, B. Sugar, S. Sumimoto, W. Tang, J. Taylor, R. Thakur, A. Trefethen, M. Valero, A. van der Steen, J. Vetter, P. Williams, R. Wisniewski, and K. Yelick. The international exascale software project roadmap. International Journal of High Performance Computing Applications, 25(1):3-60, 2011.

[7] DoE Exascale Co-Design Center for Materials in Extreme Environments. http://www. exmatex.org, 2014.

[8] G. Faanes, A. Bataineh, D. Roweth, T. Court, E. Froese, B. Alverson, T. Johnson, J. Kopnick, M. Higgins, and J. Reinhard. Cray cascade: a scalable HPC system based on a dragonfly network. In 
Proceedings of the International Conference on High Performance Computing, Networking, Storage and Analysis, pages 1-9, Salt Lake City, Utah, 2012. IEEE Computer Society Press.

[9] M. Geimer, F. Wolf, B. J. N. Wylie, E. Ábrahám, D. Becker, and B. Mohr. The Scalasca performance toolset architecture. Concurrency and Computation: Practice and Experience, 22(6):702-719, Apr. 2010.

[10] W. Gropp, E. Lusk, and A. Skjellum. Using MPI: portable parallel programming with the message-passing interface. Scientific and engineering computation. MIT Press, Cambridge, MA, 2nd edition, 1999.

[11] W. Gropp, R. Thakur, and E. Lusk. Using MPI-2: Advanced Features of the Message Passing Interface. MIT Press, Cambridge, MA, USA, 1999.

[12] J. He, A. Snavely, R. van der Wijngaart, and M. Frumkin. Automatic recognition of performance idioms in scientific applications. In Parallel Distributed Processing Symposium (IPDPS), 2011 IEEE International, pages 118-127, May 2011.

[13] I. Karlin, A. Bhatele, J. Keasler, B. L. Chamberlain, J. Cohen, Z. DeVito, R. Haque, D. Laney, E. Luke, F. Wang, D. Richards, M. Schulz, and C. Still. Exploring traditional and emerging parallel programming models using a proxy application. In 27th IEEE International Parallel \& Distributed Processing Symposium (IEEE IPDPS 2013), Boston, USA, May 2013.

[14] I. Karlin, J. Keasler, and R. Neely. Lulesh 2.0 updates and changes. Technical Report LLNL-TR-641973, Lawrence Livermore National Laboratory, August 2013.

[15] S. Karlsson and M. Brorsson. A comparative characterization of communication patterns in applications using MPI and shared memory on an IBM SP2. In D. Panda and C. Stunkel, editors, Network-Based Parallel Computing Communication, Architecture, and Applications, volume 1362 of Lecture Notes in Computer Science, pages 189-201. Springer Berlin Heidelberg, 1998.

[16] D. J. Kerbyson and K. J. Barker. Automatic identification of application communication patterns via templates. In Proceedings of the 5th international conference on Software engineering, pages 114-121. ISCA, September 2005.

[17] J. Kim and D. J. Lilja. Characterization of communication patterns in message-passing parallel scientific application programs. In Proceedings of the Second International Workshop on Network-Based Parallel Computing: Communication, Architecture, and Applications, CANPC '98, pages 202-216, London, UK, UK, 1998. Springer-Verlag.

[18] P. Kogge, K. Bergman, S. Borkar, D. Campbell, W. Carlson, W. Dally, M. Denneau, P. Franzon, W. Harrod, K. Hill, J. Hiller, S. Karp, S. Keckler, D. Klein, R. Lucas, M. Richards, A. Scarpelli, S. Scott, A. Snavely, T. Sterling, R. S. Williams, and K. Yelick. Exascale computing study: Technology challenges in achieving exascale systems. Technical report, DARPA Information Processing Techniques Office, 2008.
[19] B. P. Miller, M. D. Callaghan, J. M. Cargille, J. K. Hollingsworth, R. B. Irvin, K. L. Karavanic, K. Kunchithapadam, and T. Newhall. The Paradyn parallel performance measurement tool. Computer, 28(11):37-46, Nov. 1995.

[20] mpiP: Lightweight, Scalable MPI Profiling. http://mpip.sourceforge.net, 2013.

[21] Nek5000. http://nek5000.mcs.anl.gov, 2014.

[22] A. Nichols. User's manual for ALE3D: An arbitrary lagrange/eulerian 3d code system. Technical Report UCRL-MA-152204 Rev 6, Lawrence Livermore National Laboratory, 2007.

[23] S. Plimpton. Fast parallel algorithms for short-range molecular dynamics. Journal of Computational Physics, 117:1-19, 1995.

[24] P. G. Raponi, F. Petrini, R. Walkup, and F. Checconi. Characterization of the communication patterns of scientific applications on blue gene/p. In Parallel and Distributed Processing Workshops and Phd Forum (IPDPSW), 2011 IEEE International Symposium on, pages 1017-1024, May 2011.

[25] J. Shalf, S. Kamil, L. Oliker, and D. Skinner. Analyzing ultra-scale application communication requirements for a reconfigurable hybrid interconnect. In 2005 ACM/IEEE conference on Supercomputing, page 17. IEEE Computer Society, 2005.

[26] S. Shende and A. Malony. The TAU parallel performance system. International Journal of High Performance Computing Applications, 20(2):287-311, 2006.

[27] The NumPy Community. NumPy reference, release 1.8.1, March 2014.

[28] The SciPy Community. SciPy reference guide, release 0.13.0, October 2013.

[29] J. Vetter, R. Glassbrook, J. Dongarra, K. Schwan, B. Loftis, S. McNally, J. Meredith, J. Rogers, P. Roth, K. Spafford, and S. Yalamanchili. Keeneland: Bringing heterogeneous GPU computing to the computational science community. Computing in Science Engineering, 13(5):90-95, 2011.

[30] J. S. Vetter, editor. Contemporary High Performance Computing: From Petascale Toward Exascale, volume 1 of CRC Computational Science Series. Taylor and Francis, Boca Raton, 1 edition, 2013.

[31] J. S. Vetter, S. Lee, D. Li, G. Marin, C. McCurdy, J. Meredith, P. C. Roth, and K. Spafford. Quantifying architectural requirements of contemporary extreme-scale scientific applications. In International Workshop on Performance Modeling, Benchmarking and Simulation of HPC Systems (PMBS13), Denver, Colorado, 2013.

[32] J. S. Vetter and M. O. McCracken. Statistical scalability analysis of communication operations in distributed applications. SIGPLAN Not., 36(7):123-132, June 2001.

[33] J. S. Vetter and F. Mueller. Communication characteristics of large-scale scientific applications for contemporary cluster architectures. Journal of Parallel and Distributed Computing, 63(9):853-865, 2003. 